\title{
Observações de onda de gravidade e bolha de plasma na ionosfera equatorial, sobre a Amazônia Central (Manaus-Brasil) em 2015, utilizando imageador All-Sky.
}

\author{
'LIMA, N*., 'FERREIRA, A., ' ${ }^{1}$ SILVA, R., ${ }^{1}$ GÓES, K., ${ }^{1}$ FARIAS, T., ${ }^{1}$ ARAÚJO, Y., ${ }^{1}$ CHARLESTIN, W., ${ }^{1}$ REIS, W. \\ (1) Centro Universitário Luterano de Manaus - CEULM/ULBRA \\ Av. Carlos Drummond de Andrade, 1460. Conjunto Atílio Andreazza. Bairro Japiim II. \\ Manaus-AM/Brasil. CEP. 69077-730. Tel. (92) 3616-9800. \\ (e-mail:newtonulbra@gmail.com)
}

Copyright 2016, SBGf - Sociedade Brasileira de Geofísica

Este texto foi preparado para a apresentação no VII Simpósio Brasileiro de Geofísica, Ouro Preto, 25 a 27 de outubro de 2016. Seu conteúdo foi revisado pelo Comité Técnico do VII SimBGf, mas não necessariamente representa a opinião da SBGf ou de seus associados. E proibida a reprodução total ou parcial deste material para propósitos comerciais sem prévia autorização da SBGf.

\section{Resumo}

Estudo de observação sobre onda de gravidade e o processo de bolhas de plasma na ionosfera tropical, na Amazônia Central no setor longitudinal brasileiro. Desta forma, este trabalho irá contribuir para uma melhor compreensão da dinâmica das bolhas de plasma na região tropical, durante períodos de atividade geomagnética calma e perturbada. Esse estudo foi realizado através de fotômetro imageadores tipo "all-sky", instalados na Reserva Biológica do Rio Cuieiras - ZF-2 BR 174 (km 50 ramal 34 km (ao norte de Manaus-AM). Os resultados mostram a eficiência do equipamento para - monitoramento proposto aos fenômenos estudos próximo do equinócio (agosto de 2015).

\section{Introdução}

Apresentado em Wrasse et al., 2007, que as observações da aeroluminescência noturna através de imageadores tem sido usada para investigar a estrutura horizontal das ondas de gravidade, bem como para observar as características das ondas de curto período e pequeno comprimento de onda horizontal (5 100 km). Também em Wrasse et al., 2007, somente as ondas com comprimentos de onda vertical maiores que a largura característica das camadas de emissão são possíveis de serem detectadas.

O fotômetro imageador all-sky mede a distribuição espacial de uma luz muito tênue que é emitida pela atmosfera terrestre. Essa luz é chamada de luminescência Atmosférica (PIMENTA, 2003).

A luminescência atmosférica de uma transição atômica ou molecular especifica ocorre em uma faixa estreita da atmosfera. E a observação da distribuição espacial da luminescência fornece informações sobre a região da atmosfera onde se originou a mesma (SAHAl et al., 2000).

Reações químicas que ocorrem na média e alta atmosfera dão origem a átomos ou moléculas excitadas (nível de energia superior ao seu estado fundamental). $\mathrm{O}$ átomo ou molécula excitado ao decair para um nível de energia menor ou para o estado fundamental emite um fóton. Os fótons são emitidos em todas as direções e uma parcela destes fótons é emitida na direção da terra e o fotômetro imaginador mede esses fótons (SAHAl et al., 1994).

\section{Metodologia}

Para as observações realizadas dentro do período desta pesquisa foi utilizado um imageador tipo All-Sky (Fig. 01) e os filtros necessários para aquisição das imagens dentro do comprimento especificado (Tab. 01), são usados ao longo da trajetória ótica que é interrompida por esta roda de filtros composta de cinco filtros de interferência, para o Ol 630,0 nm, para o fundo luminoso em $578,0 \mathrm{~nm}$, um filtro passa banda colorido no infravermelho entre 715 e $930 \mathrm{~nm}$ (bandas (8,3), (4,0), $(9,4),(5,1),(6,2),(7,3)$ e parte da $(8,4)$ do $\mathrm{OH})$ sem transmissão em $865 \mathrm{~nm}$ e um para a banda do $02(0,1)$ em 865 nm, (WRASSE, 2007), equipamento confinado em um container no sítio na Reserva Biológica do Cuieiras - ZF-2 - BR 174 (km 50- ramal 34 km) ao norte de Manaus-AM). O período das observações ocorreu entre agosto e setembro de 2015, estação seca na Amazônia. As observações foram realizadas durante período de lua nova. As imagens adquiridas foram de onda de gravidade (Fig. 02) e bolhas de plasma (Fig. 03). 

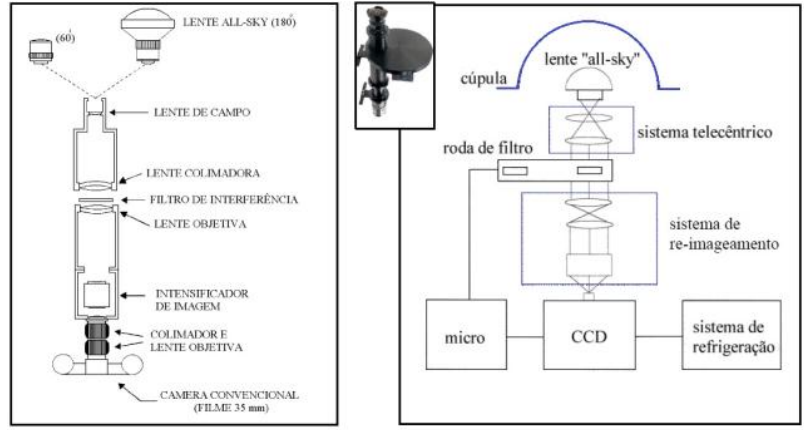

Fig. 01: Esquema do Equipamento - Fotômetro imageador ALL SKY, (PIMENTA, 2003).

Tabela 01: Características dos filtros e alturas médias das camadas de luminescência do oxigênio atômico. (Segundo, Pimenta, 2003).

\begin{tabular}{|c|c|c|}
\hline $\begin{array}{c}\text { Comprimento de onda } \\
\text { das emissões OI }(\mathrm{nm})\end{array}$ & $\begin{array}{c}\text { Largura de banda } \\
(\mathrm{nm})\end{array}$ & $\begin{array}{c}\text { Altura média da } \\
\text { camada }(\mathrm{km})\end{array}$ \\
\hline 630,0 & 1,5 & $250-300$ \\
\hline 557,7 & 2,65 & $250-300$ \\
\hline 777,4 & 1,35 & $300-400$ \\
\hline
\end{tabular}

\section{Resultados e Discussão}

Observa-se na Fig. 02 deslocamento de ondas de gravidade sobre o alvo do estudo em 18 de Agosto de 2015 , no intervalo de aproximadamente $1,5 \mathrm{~h}$, durante 0 período de aquisição das imagens a magnetosfera terrestre encontrava-se em fase de recuperação de seu estado neutro após uma dst (disturbance storm time) de intensidade -84 nT, entre $07 \mathrm{~h}$ e $08 \mathrm{~h}$ UT (WDC for Geomagnetism, Kyoto, 2015). A formação de bolhas de plasma sobre a Amazônia Central é evidenciada na Fig. 03, que mostra a dinâmica da evolução deste fenômeno de grande escala para o dia 19 de Agosto de 2015, neste dia ocorreu um dst de intensidade $-51 \mathrm{nT}$, (WDC for Geomagnetism, Kyoto, 2015) assim como geração de "spread $F$ " sobre o alvo é mostrado na aquisição do VTEC - Vertical Total Electron Content (GPS) na estação de Manaus (ULBRA-UNIVAP) (Fig. 04). No intervalo de 061623 UT até 081019 UT, dentro do espectro de emissões do IO 630,0, com largura de faixa $1,5 \mathrm{~nm}$, em $300 \mathrm{~km}$ de altura é mostrado o fenômeno de bolha de plasma na Fig. 03. Os dados destes eventos dos dias 18 e 19 de Agosto de 2015 foram correlacionados com informação de magnetrômetros e GPS, Fig. 04 (Índice DST e VTEC), que contribuíram para comprovação de instabilidades do plasma ionosférico (Região F) nestes dias.

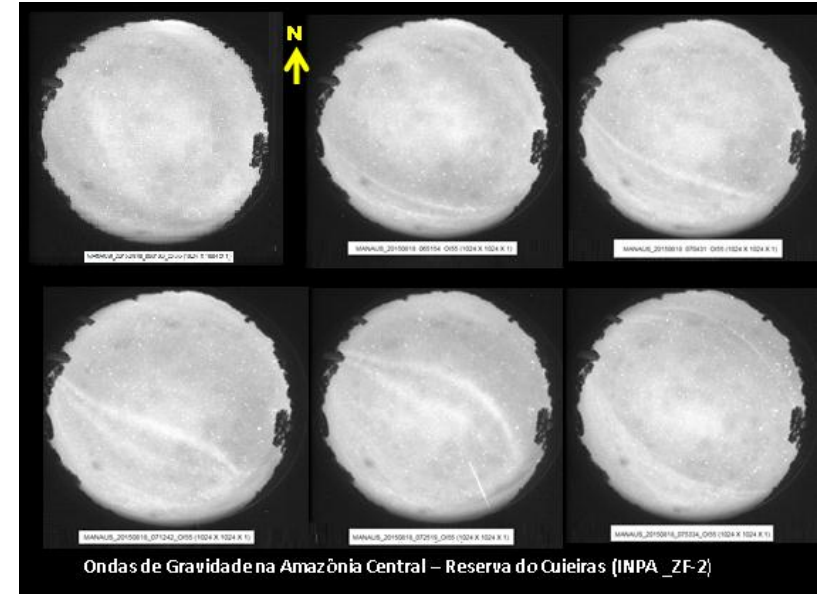

Fig. 02: Painel de imagens por aeroluminescência da assinatura de ondas de gravidade sobre a Amazônia Central próximo do equinócio vernal (18 Agosto 2015) em período geomagnético calmo, imagens no intervalo de 06 3106 UT até 075334 UT, área de 1024 km x 1024 km, dentro do espectro do Ol 557,7, com largura de banda $6,25 \mathrm{~nm}$, em aproximadamente $300 \mathrm{~km}$ de altura. Cortesia IP\&D - UNIVAP, 2015.

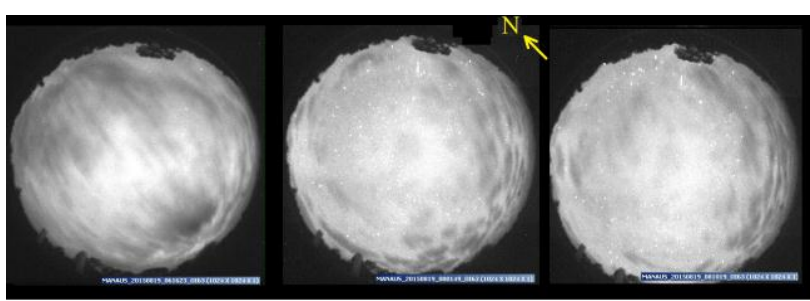

Fig. 03: Painel de imagens por aeroluminescência da evolução na dinâmica da formação de Bolha de Plasma em 19 de Agosto de 2015 entre 061623 UT até 081019 UT, no comprimento de onda das emissões Ol 630,0, com largura de banda de $1,5 \mathrm{~nm}$, área de $1024 \mathrm{~km} \mathrm{x}$ 1024 km. Cortesia IP\&D - UNIVAP, 2015.

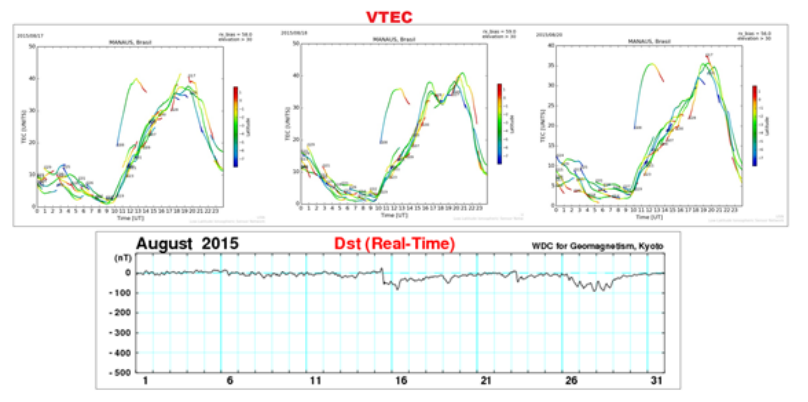

Fig. 04: Painel dos Índices VTEC (Manaus-Brasil) para os dias 17, 18 e 20 do mês de Agosto de 2015 e DST (todo o mês de Agosto 2015). Cortesia LISN, WDC-Kyoto, 2015. 
O fenômeno de bolha de plasma é também observado nos receptores GPS na área deste estudo (Fig. 05), quando em aquisição de posicionamento na região durante a noite são notados riscos nas telas dos receptores nos horários estudados.

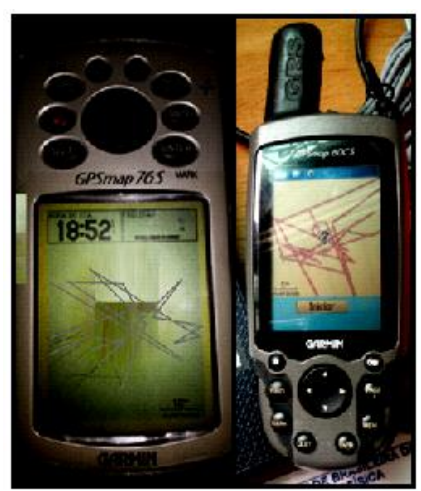

Fig. 05: Telas dos receptores GPS em posicionamento estacionário registrando o sinal GNSS (Global Navigation Satellite System) em múltiplos traçados.

\section{Conclusões}

Com auxílio do Imageador All-Sky, dentro do período lunar adequado é possível monitorar fenômenos de grande escala na lonosfera (região F) da Mesosfera e Termosfera na região equatorial na Amazônia Central, visto que existe seres humanos trafegando na altitude deste estudo, (Estação Espacial Internacional).

\section{Agradecimentos}

Os autores são gratos ao Edital 030./2013 (UNIVERSAL AMAZONAS-FAPEAM) ao IP\&D-UNIVAP/SP, ao INPA, a LISN e a WDC-Kyoto, ao CEULM-ULBRA, pelas informações e cortesia de imagens deste trabalho.

\section{Referências}

LISN. Low Latitude Ionospheric Sensor Network. Disponível em: <http://www.lisn.igp.gov.pe>, acesso: 17, 18 e 20 de agosto de 2015.

PIMENTA, A. A.: Tese de Doutorado (INPE-2003), 196 p.

SAHAI, Y.; AARONS, J.; BAUMGARDNER, J.; BITTENCOURT, J.A.; TAKAHASHI, H. Ol $630 \mathrm{~nm}$ imaging observations of the equatorial plasma depletions at $16^{\circ} \mathrm{S}$ dip latitude. Journal of Atmospheric and Terrestrial Physics, v.56, no.11, p. 1461-1475, 1994.

SAHAI, Y.; FAGUNDES, P.R.; BITTENCOURT, J.A. Transequatorial F-region ionospheric plasma bubbles: solar cycle effects. Journal of Atmospheric and Terrestrial Physics, v.62, no.15, p. 1377-1383, 2000.
WDC for Geomagnetism. WORLD DATA CENTER. Kyoto University, 2015. Disponível em: $<$ http://wdc.kugi.kyotoac.jp/dst_realtime/201508/index.ht $\mathrm{ml}>$, Acesso em 08, 2015.

WRASSE, Cristiano Max, TAKAHASHI, Hisao, MEDEIROS, Amauri Fragoso de, LIMA, Lourivaldo Mota, TAYLOR, Michael John, GOBBI, Delano e FECHINE, Joaquim. DETERMINAÇÃO DOS PARÂMETROS DE ONDAS DE GRAVIDẢDE ATRAVÉS DA ANÁLISE ESPECTRAL DE IMAGENS DE AEROLUMINESCÊNCIA. Revista Brasileira de Geofísica (2007) 25(3): 257 266. Sociedade Brasileira de Geofísica ISSN 0102-261X. 2007. 